PROCEEDINGS OF THE

AMERICAN MATHEMATICAL SOCIETY

Volume 128, Number 9, Pages 2513-2516

S 0002-9939(00)05879-2

Article electronically published on April 27, 2000

\title{
A NOTE ON TENSOR PRODUCTS OF AMPLE LINE BUNDLES ON ABELIAN VARIETIES
}

\author{
YOSHIAKI FUKUMA
}

(Communicated by Ron Donagi)

\begin{abstract}
Let $(X, L)$ be a polarized abelian variety defined over the complex number field. Then we classify $(X, L)$ with $h^{0}(L) \geq 2$ such that $(k+1) L$ is not $k$-jet ample nor $k$-very ample.
\end{abstract}

\section{Introduction}

Let $X$ be an abelian variety defined over the complex number field, and let $L_{1}, \ldots, L_{t}$ be ample line bundles on $X$. Then, in BaSz, Bauer and Szemberg proved that $L_{1}+\cdots+L_{k+2}$ is $k$-jet ample for $t=k+2$. In particular, if $(X, L)$ is a polarized abelian variety, then $(k+2) L$ is $k$-jet ample.

By the above result, it is natural and interesting to consider the following problem.

Problem. Let $X$ be an abelian variety defined over the complex number field, and let $L_{1}, \ldots, L_{t}$ be ample line bundles on $X$. Then characterize $\left(X, L_{1}, \ldots, L_{t}\right)$ such that $L_{1}+\cdots+L_{t}$ is not $k$-jet ample for $t \leq k+1$.

In this note, we obtain the characterization of $(X, L)$ with $h^{0}(L) \geq 2$ such that $(k+1) L$ is not $k$-jet ample nor $k$-very ample.

We work over the complex number field, and we use the customary notation in algebraic geometry.

\section{Preliminaries}

Definition 1.1. (1) (See [BeSo2.) Let $(X, L)$ be a polarized manifold. Then for a nonnegative integer $k, L$ is called $k$-jet ample if the restriction map

$$
H^{0}(L) \rightarrow H^{0}\left(L \otimes \mathcal{O}_{X} /\left(\mathfrak{m}_{y_{1}}^{k_{1}} \otimes \cdots \otimes \mathfrak{m}_{y_{r}}^{k_{r}}\right)\right)
$$

is surjective for any choice of distinct points $y_{1}, \ldots, y_{r}$ in $X$ and positive integers $k_{1}, \ldots, k_{r}$ with $\sum_{i=1}^{r} k_{i}=k+1$, where $\mathfrak{m}_{y_{i}}$ denotes the maximal ideal at $y_{i}$.

(2) (See $[\mathrm{BeSo3}]$.) Let $(X, L)$ be a polarized manifold. Then for a nonnegative integer $k, L$ is called $k$-very ample if for any 0 -dimensional subscheme $\left(Z, \mathcal{O}_{Z}\right)$ with

Received by the editors July 29, 1997.

1991 Mathematics Subject Classification. Primary 14C20.

Key words and phrases. Polarized abelian variety, $k$-jet ample, $k$-very ample.

The author is a Research Fellow of the Japan Society for the Promotion of Science. 
length $\mathcal{O}_{Z}=k+1$, the map

$$
\Gamma(L) \rightarrow \Gamma\left(L \otimes \mathcal{O}_{Z}\right)
$$

is surjective.

Remark 1.2. 1. If $L$ is $k$-jet ample, then $L$ is $k$-very ample. (See Proposition 2.2 in [BeSo2].)

2. Let $(X, L)$ be a polarized manifold such that $L$ is $k$-jet ample (resp. $k$-very ample). Then for any smooth projective subvariety $S$ on $X, L_{S}$ is $k$-jet ample (resp. $k$-very ample).

Theorem 1.3 (Bauer-Szemberg). Let $X$ be an abelian variety, and let $L_{1}, \ldots, L_{k+1}$ be ample line bundles on $X$. Assume that $L_{k+1}$ has no fixed component. Then $L_{1}+\cdots+L_{k+1}$ is $k$-jet ample for $k \geq 1$.

Proof. See Theorem 2.1 in $\mathrm{BaSz}$.

Lemma 1.4 (Ohbuchi). Let $X$ be an abelian variety with $\operatorname{dim} X=n \geq 2$, and let $L$ be an ample line bundle on $X$. Let $|M|$ be the movable part of $|L|$, and let $F$ be the fixed part of $|L|$. If $h^{0}(L) \geq 2$ and $M^{n}=0$, then

$$
(X, L) \cong\left(A_{1} \times A_{2}, p_{1}^{*}\left(D_{1}\right)+p_{2}^{*}\left(D_{2}\right)\right),
$$

where $A_{1}$ and $A_{2}$ are abelian varieties, $D_{i}$ is an ample divisor on $A_{i}$ for $i=1,2$ with $h^{0}\left(D_{1}\right)=1$, and $p_{i}$ is the $i$-th projection $A_{1} \times A_{2} \rightarrow A_{i}$ for $i=1,2$.

Proof. See Lemma 4 in $\mathrm{O}$.

Lemma 1.5. Let $C$ be a smooth elliptic curve, and let $D$ be an ample divisor on $C$ with $\operatorname{deg} D \geq 2$. Then $(k+1) D$ is $k$-jet ample.

Proof. We remark that for a line bundle on a smooth projective curve, the notions " $k$-jet ample", " $k$-very ample", and " $k$-spanned" coincide. (See Proposition 2.1 in [BeSo2].)

If $k=0$, then $D$ is spanned since $\operatorname{deg} D \geq 2$.

If $k=1$, then $D$ is very ample since $2 \operatorname{deg} D \geq 4=2 g(C)+2$.

If $k \geq 2$, then $D$ is $k$-jet ample by Lemma 1.1 in [BeSo1].

Lemma 1.6. Let $X_{1}$ and $X_{2}$ be smooth projective varieties, and let $L_{1}$ and $L_{2}$ be line bundles on $X_{1}$ and $X_{2}$ respectively. For $i=1,2$, assume that $L_{i}$ is $k_{i}$-jet ample (resp. $k_{i}$-very ample) and let $k:=\min \left\{k_{1}, k_{2}\right\}$. Then $p_{1}^{*}\left(L_{1}\right)+p_{2}^{*}\left(L_{2}\right)$ is $k$-jet ample (resp. $k$-very ample), where $p_{i}$ is the $i$-th projection $X_{1} \times X_{2} \rightarrow X_{i}$ for $i=1,2$.

Proof. See Lemma 1.8 in [BeDiSo, and Lemma 3.3 in BeSo4].

\section{MAin THEOREMS}

Theorem 2.1. Let $(X, L)$ be a polarized abelian variety with $\operatorname{dim} X=n \geq 2$ and $h^{0}(L) \geq 2$. Then for $k \geq 1,(k+1) L$ is not $k$-jet ample if and only if $(X, L)$ is the following (\$):

(4) $X \cong A_{1} \times \cdots \times A_{m}$ and $L=p_{1}^{*}\left(D_{1}\right)+\cdots+p_{m}^{*}\left(D_{m}\right)$, where each $A_{i}$ is an abelian variety, $D_{i}$ is an ample divisor on $A_{i}$ with $h^{0}\left(D_{i}\right)=1$ for $i=1, \ldots, m-1$ and $h^{0}\left(D_{m}\right) \geq 2$, and for some $j(\neq m),(k+1) D_{j}$ is not $k$-jet ample. 
Proof. First we prove the "if" part. Assume that $(k+1) L$ is $k$-jet ample and $(X, L)$ is the type $(\boldsymbol{\beta})$. By assumption, $(k+1) D_{j}$ is not $k$-jet ample. Let $F_{t}$ be a fiber of the $t$-th projection of $A_{1} \times \cdots \times A_{m}$. Then

$$
\bigcap_{t \neq j} F_{t} \cong A_{j}
$$

and

$$
L_{A_{j}}=D_{j}
$$

Since $(k+1) L$ is $k$-jet ample, we obtain that $(k+1) L_{A_{j}}$ is also $k$-jet ample. But this is a contradiction because $L_{A_{j}}=D_{j}$.

Next we prove the "only if" part. Assume that $(k+1) L$ is not $k$-jet ample. By Theorem 1.3, we get $\operatorname{dim} \operatorname{Bs}|L|=n-1$. Let $|M|$ be the movable part of $|L|$, and let $F$ be the fixed part of $|L|$.

Assume that $M^{n}>0$. Then $M$ is ample. We remark that $F$ is nef since $F$ is an effective divisor on an abelian variety $X$. Hence $L+F$ is ample. On the other hand

$$
(k+1) L=(L+F)+\underbrace{L+\cdots+L}_{k-1}+M .
$$

Since $\operatorname{dim} \operatorname{Bs}|M|<n-1$, we get that $(k+1) L$ is $k$-jet ample by Theorem 1.3. But this is a contradiction by assumption.

Hence $M^{n}=0$. By Lemma 1.4, we get that $X \cong A_{1} \times X_{1}$ and $L=p_{A, 1}^{*}\left(D_{1}\right)+$ $p_{X, 1}^{*}\left(D_{X, 1}\right)$, where $A_{1}$ and $X_{1}$ are abelian varieties, $p_{A, 1}$ is the projection $X \rightarrow A_{1}$, $p_{X, 1}$ is the projection $X \rightarrow X_{1}, D_{1}$ is an ample divisor on $A_{1}$ and $D_{X, 1}$ is an ample divisor on $X_{1}$ with $h^{0}\left(D_{1}\right)=1$ and $h^{0}\left(D_{X, 1}\right) \geq 2$.

If $(k+1) D_{1}$ is not $k$-jet ample, then this completes the proof by putting $A_{2}:=X_{1}$, $p_{1}:=p_{A, 1}, p_{2}:=p_{X, 1}$, and $D_{2}:=D_{X, 1}$.

If $(k+1) D_{1}$ is $k$-jet ample, then $(k+1) D_{X, 1}$ is not $k$-jet ample by Lemma 1.6. In this case, we put $p_{1}:=p_{A, 1}$ and we go to the following step for $i=1$, where $L_{0}:=L$.

Step (i). Let $L_{i}:=\left.L_{i-1}\right|_{X_{i}}$. Then $L_{i}=D_{X, i}$. Assume that $(k+1) L_{i}$ is not $k$-jet ample. Let $\left|M_{i}\right|$ be the movable part of $\left|L_{i}\right|$, and let $F_{i}$ be the fixed part of $\left|L_{i}\right|$. Then by the same argument as above, we can prove that $F_{i} \neq 0$ and $M_{i}^{n_{i}}=0$, where $n_{i}=\operatorname{dim} X_{i}$. Hence by Lemma 1.4, we get that $X_{i} \cong A_{i+1} \times X_{i+1}$ and $L_{i}=p_{A, i+1}^{*}\left(D_{i+1}\right)+p_{X, i+1}^{*}\left(D_{X, i+1}\right)$, where $A_{i+1}$ and $X_{i+1}$ are abelian varieties, $p_{A, i+1}$ is the projection $X_{i} \rightarrow A_{i+1}, p_{X, i+1}$ is the projection $X_{i} \rightarrow X_{i+1}, D_{i+1}$ is an ample divisor on $A_{i+1}$ and $D_{X, i+1}$ is an ample divisor on $X_{i+1}$ with $h^{0}\left(D_{i+1}\right)=1$ and $h^{0}\left(D_{X, i+1}\right) \geq 2$.

If $(k+1) D_{i+1}$ is not $k$-jet ample, then this completes the proof by putting $A_{i+2}:=X_{i+1}, p_{i+1}:=p_{A, i+1} \circ p_{X, i} \circ \cdots \circ p_{X, 1}, p_{i+2}:=p_{X, i+1} \circ p_{X, i} \circ \cdots \circ p_{X, 1}$, and $D_{i+2}:=D_{X, i+1}$.

If $(k+1) D_{i+1}$ is $k$-jet ample, then $(k+1) D_{X, i+1}$ is not $k$-jet ample by Lemma 1.6. In this case, we put $p_{i+1}:=p_{A, i+1} \circ p_{X, i} \circ \cdots \circ p_{X, 1}$ and we go to Step $(i+1)$.

The above procedures come to an end after a finite number of repetitions by Lemma 1.5. This completes the proof of Theorem 2.1.

By using Theorem 1.3 and Lemma 1.4, we can prove the following by an argument similar to that in the proof of Theorem 2.1. 
Theorem 2.2. Let $X$ be an abelian variety, and let $L_{1}, \ldots, L_{k+1}$ be ample line bundles on $X$ for $k \geq 1$ with $h^{0}\left(L_{i}\right) \geq 2$ for some $i$. If $L_{1}+\cdots+L_{k+1}$ is not $k$-jet ample, then $X \cong A_{1} \times A_{2}$, where $A_{1}$ and $A_{2}$ are abelian varieties.

We can also prove the following theorem by the same argument as in the proof of Theorem 2.1 and Theorem 2.2.

Theorem 2.3. (1) Let $(X, L)$ be a polarized abelian variety with $\operatorname{dim} X=n \geq 2$ and $h^{0}(L) \geq 2$. Then for $k \geq 1,(k+1) L$ is not $k$-very ample if and only if $(X, L)$ is the following (\$):

(a) $X \cong A_{1} \times \cdots \times A_{m}$ and $L=p_{1}^{*}\left(D_{1}\right)+\cdots+p_{m}^{*}\left(D_{m}\right)$, where each $A_{i}$ is an abelian variety, $D_{i}$ is an ample divisor on $A_{i}$ with $h^{0}\left(D_{i}\right)=1$ for $i=1, \ldots, m-1$ and $h^{0}\left(D_{m}\right) \geq 2$, and for some $j(\neq m),(k+1) D_{j}$ is not $k$-very ample.

(2) Let $X$ be an abelian variety, and let $L_{1}, \ldots, L_{k+1}$ be ample line bundles on $X$ for $k \geq 1$ with $h^{0}\left(L_{i}\right) \geq 2$ for some $i$. If $L_{1}+\cdots+L_{k+1}$ is not $k$-very ample, then $X \cong A_{1} \times A_{2}$, where $A_{1}$ and $A_{2}$ are abelian varieties.

\section{REFERENCES}

[BaSz] Th. Bauer and T. Szemberg, Higher order embeddings of abelian varieties, Math. Z. 224 (1997), 449-455. MR 98a:14009

[BeDiSo] M. C. Beltrametti, S. Di Rocco, and A. J. Sommese, On higher order embeddings of Fano threefolds by the anticanonical linear system, J. Math. Sci. Univ. Tokyo 5 (1998), 75-97. MR 99d:14036

[BeSo1] M. C. Beltrametti and A. J. Sommese, On k-spannedness for projective surfaces, In Algebraic Geometry (L'Aquila, 1988) Lecture Notes in Math. 1417 (1990), 24-51. MR 91g:14029

[BeSo2] M. C. Beltrametti and A. J. Sommese, On k-jet ampleness, In Complex Analysis and Geometry, editted by V. Ancona and A. Silva, Plenum Press, New York (1993), 335-376. MR 94g:14006

[BeSo3] M. C. Beltrametti and A. J. Sommese, The adjunction theory of complex projective varieties, de Gruyter Expositions in Math. 16 (1995). MR 96f:14004

[BeSo4] M. C. Beltrametti and A. J. Sommese, Notes on Embeddings of blowups, J. Algebra 186 (1996), 861-871. MR 97m:14004

[O] A. Ohbuchi, Some remarks on ample line bundles on abelian varieties, Manuscripta Math. 57 (1987), 225-238. MR 87m:14051

Department of Mathematics, Faculty of Science, Tokyo Institute of Technology, Oh-OKayama, Meguro-Ku, TOKYo 152-8551, Japan

E-mail address: fukuma@math.titech.ac.jp

Current address: Department of Mathematics, Naruto University of Education, Takashima, Naruto-cho, Naruto-shi 772-8502, Japan

E-mail address: fukuma@naruto-u.ac.jp 